\title{
Paediatric Rhegmatogenous Retinal Detachment: Clinical Features and Surgical Outcomes
}

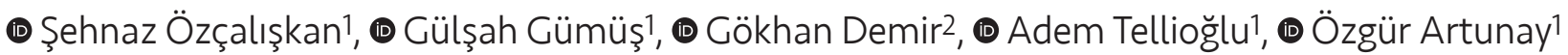 \\ 1University of Health Sciences Turkey, Beyoğlu Eye Training and Research Hospital, Clinic of Ophthalmology, İstanbul, Turkey \\ 2University of Health Sciences Turkey, Fatih Sultan Mehmet Training and Research Hospital, Clinic of Ophthalmology, İstanbul, Turkey
}

\begin{abstract}
Aim: To evaluate the clinical characteristics, predisposing factors and surgical outcomes of rhegmatogenous retinal detachment (RRD) in paediatric patients.

Materials and Methods: This retrospective consecutive case series included 79 paediatric patients diagnosed with RRD who were treated by surgical intervention. Demographic data, predisposing factors, the status of macular involvement, presence of proliferative vitreoretinopathy (PVR), the type of initial surgery, and the surgical anatomical and visual outcomes of the patients were recorded.

Results: The study included 79 eyes of 79 children with a mean age of $11.63 \pm 4.36$ years. There were $59(74.7 \%)$ male and 20 (25.3\%) female patients in the study group. The mean follow-up period was $18.65 \pm 6.04$ months. Trauma (45\%) was found to be the most common predisposing factor associated with RRD. Other common predisposing factors were myopia (42\%), congenital/developmental anomaly ( $9 \%)$, and history of ocular surgery (4\%). Macular detachment was present in 56 eyes (70.8\%). At the initial examination, the mean VA (VA) was $1.72 \pm 1.01$ logMAR $(n=60)$. As a primary surgical treatment, 48 eyes $(60.7 \%)$ had undergone pars plana vitrectomy (PPV), 11 eyes (13.9\%) had scleral buckle (SB), and $20(25.3 \%)$ eyes had PPV combined with SB procedures. Reattachment was achieved in $50(63.2 \%)$ eyes after the first surgery. The final mean VA was improved to $1.58 \pm 0.87 \log M A R$, and the anatomical success rate was $74.6 \%$.

Conclusion: This study demonstrated that paediatric RRD is commonly associated with trauma and myopia. Successful outcomes were obtained in both groups after RRD surgery. Although associated with poor visual outcomes, careful ophthalmologic assessment and surgical management can preserve visual functions in children with RRD.
\end{abstract}

Keywords: Pars plana vitrectomy, paediatric rhegmatogenous retinal detachment, scleral buckle

\section{Introduction}

Retinal detachment in the paediatric population is a rare clinical condition as compared to adults (1). Rhegmatogenous retinal detachment (RRD) in children constitutes 3 to 7 percent of all RRDs (2,3). Myopia, trauma, prior surgery, and congenital or developmental anomalies are defined as predisposing factors for RRD occurrence in children $(4,5)$. Previous studies have shown that clinical features, surgical outcomes, and prognosis of RRD in children differ from adults $(1,6,7)$. Systemic and ocular comorbidities, severe developmental disorders, macular involvement and proliferative vitreoretinopathy (PVR) are associated with poor prognosis in paediatric RRD (7-9).

Decreased vision is the most common complaint in all RRDs, however, the disease is often diagnosed at a later stage in the paediatric age group compared to adults (1). 
The lack of self-expression capacity and immature cognitive functions of children may cause the late diagnosis of RRD. Previous studies reported that $40-70 \%$ of patients had visual disturbances at the time of diagnosis $(10,11)$. Owing to late diagnosis, paediatric patients are more likely to present with a higher percentage of macular involvement and PVR $(4,11)$. Despite improvements in instruments and surgical techniques, paediatric RRD treatment remains a challenge for surgeons. In this study, we presented the clinical features and surgical outcomes of paediatric RRD patients from our tertiary referral centre. Herein, we also aim to provide contemporary data to prevent and detect this socially significant condition earlier.

\section{Materials and Methods}

We retrospectively reviewed the charts of paediatric patients (age $<18$ years) treated for a diagnosis of RRD at Beyoglu Eye Training and Research Hospital from February 2015 to April 2018. The study was approved by the Hamidiye Ethics Committee of the University of Health Sciences and conducted in adherence to the tenets of the Declaration of Helsinki. The exclusion criteria were active retinopathy of prematurity, retinoblastoma, persistent foetal vasculature, or a follow-up period of less than six months.

Age, gender, laterality, etiology, macular status, refractive status, presence of PVR (grade C or worse), presenting and final (VA), duration of follow-up, and anatomic success after initial surgery and at the end of follow-up were recorded. Patients were divided into groups according to predisposing factors as; the myopia group consisting of patients with a refractive error greater than -4 diopters, the traumatic group (history of open and closed globe injury), the congenital/developmental anomaly group consisting of patients presenting with ocular structural and developmental anomalies and the previous ocular surgery group consisting of patients with prior ocular surgery history.

Data related to operations were recorded, including the type of initial surgical procedure, endotamponade use, and the overall number of surgical interventions, including silicone oil removal procedures. Data of the initial surgery were recorded as follows; the scleral buckle (SB), pars plana vitrectomy (PPV), or combined procedure (both SB and PPV). Scleral buckling procedures were carried out in patients with visible retinal breaks. PPV was performed in patients with obscure ocular media, PVR, giant tears, undetected or multiple retinal breaks, and complicated retinal detachment. Anatomical success was defined as a retinal attachment for at least six months after primary surgery (without tamponade), and final anatomical success was defined as the complete retinal reattachment (without tamponade) at the end of the follow-up period.

\section{Statistical Analysis}

Statistical analysis was performed using SPSS software (version 22, SPSS Inc., Chicago, IL). Descriptive statistics were used to evaluate epidemiologic data, type of surgery, number of operations, and final anatomic success. Data were analysed using Wilcoxon signed-rank, KruskalWallis and Dunn tests. Logistic regression was used to assess anatomic outcomes with etiology, age, initial procedure, macular involvement and PVR> grade $C$ status. A p-value $<0.05$ was considered statistically significant.

\section{Results}

Seventy-nine eyes of 79 patients were enrolled with a mean age of $11.63 \pm 4.36$ years (range: $2-17$ ) in the study. There were $59(74.7 \%)$ males and 20 (25.3\%) females in the study group. The laterality of eyes was 33 (41.3\%) for right, and $47(58.8 \%)$ for left eyes. The mean follow-up period was $18.65 \pm 6.04$ months. Trauma was the leading etiological factor in the study group (45\%). Other factors contributing to RRD were recorded as myopia in 33 (42\%) eyes, congenital and developmental anomalies in 7 (9\%) eyes, and previous ocular surgery in $3(4 \%)$ eyes. The demographic and clinical features in the four etiologic groups are presented in Table I. Previous surgical procedures and congenital and developmental disorders are listed in Table II.

At presentation, VA was recorded in 60 (76\%) eyes. The mean VA before initial surgery was 1.72 $\pm 1.01 \log M A R$, and the macula was detached in 56 (70\%) eyes. At the end of follow-up, the mean VA was available for 69 (87\%) patients, with a mean of $1.58 \pm 0.87 \log M A R$. VAwas significantly improved after surgical interventions in the overall study group ( $p=0.042)$. In 30 (38.0\%) eyes, the grade of PVR was recorded as $\geq$ grade $C$. The details of surgical interventions, visual and outcomes in the four main groups are presented in Table III. The mean number of surgeries required for favourable anatomic success was $1.63 \pm 0.87$, with silicone oil removal procedures included. As primary surgery, 48 $(60.7 \%)$ eyes had undergone PPV, 11 (13.9\%) eyes had SB, and 20 (25.3\%) eyes had PPV combined with scleral buckling. Air was used in 2 (3\%) eyes as endotamponade. In 8 (12.9\%) eyes, perfluoropropane (C3F8) and in 6 (6.4\%) eyes, sulphuehexafluoride (SF6) gases were used for tamponade. The most commonly used vitreous substitute was silicone oil in those patients undergoing PPV. Eight (12.9\%) eyes had 1,000 centistoke (cst) silicone oil, and 38 (61.2\%) eyes had 5,000 cst silicone oil for tamponade effect. 
After primary surgery, the myopic group had the highest anatomic success rate $(66.7 \%)$. The retina was attached in 22 eyes in the myopia group, 2 (66.6\%) patients in the previous ocular surgery group, 22 (61.1\%) eyes in the trauma group, and 4 (57.1\%) eyes in the congenital/developmental anomaly group. At the end of the follow-ups, the overall

Table I. Ocular characteristics of the four main study groups

\begin{tabular}{|l|l|l|l|l|l|}
\hline & All & Trauma & Myopia & $\begin{array}{l}\text { Congenital/developmental } \\
\text { disorder }\end{array}$ & $\begin{array}{l}\text { Previous ocular } \\
\text { surgery }\end{array}$ \\
\hline Eyes (n) & 79 & $36(45.0)$ & $33(42.0)$ & $7(9.0)$ & $3(4.0)$ \\
\hline Mean age, years (SD) & $11.63(4.36)$ & $11.2(4.45)$ & 13.6 & 6.8 & 7.8 \\
\hline Male gender [n; (\%)] & $59(74.7)$ & $27(75.0)$ & $24(72.7)$ & $5(71.4)$ & $3(100)$ \\
\hline Laterality, left eyes [n; (\%)] & $47(59.4)$ & $21(58.3)$ & $21(63.7)$ & $3(42.9)$ & $2(66.7)$ \\
\hline Macula off [n; (\%)] & $56(70.8)$ & $26(72.2)$ & $23(69.7)$ & $5(71.4)$ & $3(100)$ \\
\hline $\begin{array}{l}\text { Presence of PVR > grade C } \\
\text { [n; (\%)] }\end{array}$ & $30(38.0)$ & $13(39.3)$ & $11(33.3)$ & $4(57.1)$ & $2(66.7)$ \\
\hline $\begin{array}{l}\text { Mean follow-up, months } \\
\text { (SD) }\end{array}$ & $18.2(6.40)$ & $18.4(7.03)$ & $18.8(8.50)$ & $15.8(5.98)$ & $16.7(4.04)$ \\
\hline SD: Stan) &
\end{tabular}

SD: Standard deviation, PVR: Proliferative vitreoretinopathy

Table II. Predisposing congenital/developmental ocular disorders and previous opthalmic surgeries

Predisposing factor

No of eyes

Congenital/developmental disorder $(n=7)$

Coats' disease

Von hippel lindau

Optic pit

Stargardt disease

Coloboma

Familial exudative vitreoretinopathy

Morning glory

Previous ocular surgery $(n=3)$

Cataract surgery

Glaucoma surgery

Scleral fixation of intraocular lens

Table III. Summary of surgical approach and outcomes according to predisposing factors

\begin{tabular}{|l|l|l|l|l|l|}
\hline & $\begin{array}{l}\text { All } \\
\mathbf{n = 7 9}\end{array}$ & $\begin{array}{l}\text { Trauma } \\
\mathbf{n = 3 6}\end{array}$ & $\begin{array}{l}\text { Myopia } \\
\mathbf{n = 3 3}\end{array}$ & $\begin{array}{l}\text { Congenital/developmental } \\
\text { disorder } \mathbf{n = 7}\end{array}$ & $\begin{array}{l}\text { Previous ocular } \\
\text { surgery } \mathbf{n = 3}\end{array}$ \\
\hline Initial surgery [n; (\%)] & & & & & \\
\hline Scleral buckle & $11(14)$ & $3(8.3)$ & $8(24.2)$ & - & - \\
\hline Pars plana vitrectomy & $48(61)$ & $23(63.8)$ & $17(51.5)$ & 5 & 3 \\
\hline Combined & $20(25)$ & $10(27.8)$ & $8(24.2)$ & 2 & - \\
\hline Anatomic success [n; (\%)] & $50(63.2)$ & $22(61)$ & $22(67)$ & $4(57.1)$ & $2(66.6)$ \\
\hline Mean no operations (SD) & $2.21(0.87)$ & $2.22(0.86)$ & $1.90(0.91)$ & $2.00(0.82)$ & $2.66(1.15)$ \\
\hline Final anatomic success [n; (\%)] & $59(74.6)$ & $27(75.0)$ & $25(78.7)$ & $5(71.4)$ & $2(66.6)$ \\
\hline SD: Standard deviation & & & & \\
\hline
\end{tabular}


final anatomic success rate was $74.6 \%$. Final VA was available for 69 patients with a mean $1.58 \pm 0.87 \log$ MAR. The congenital/developmental disorder and previous ocular surgery groups were combined as a miscellaneous group for further statistical analysis. The anatomic outcome after initial surgery showed no significant differences among the trauma, myopia and miscellaneous groups $(p=0.873)$. However, a significant difference was observed with regard to final anatomic success among the three groups $(p=0.035)$. A post hoc test revealed significant difference in final anatomic success between the myopia and both the trauma and miscellaneous groups $(p=0.031, p=0.021$ respectively). A logistic regression model revealed that poor final anatomic outcome predictors were etiologies other than trauma and myopia $(p=0.001)$, young age $(p=0.010)$ and macular involvement $(p<0.001)$. The anatomic outcomes were also evaluated based on initial surgical operation and no significant difference was observed in primary and final anatomic outcomes in the trauma and myopia groups with regard to initial surgery type $(p=0.374, p=0.425)$.

\section{Discussion}

In the current study, we described the etiological factors and surgical outcomes of RRD in the paediatric age group referred to our tertiary centre. Trauma is the leading etiological factor, followed by myopia in the study group. The children in the congenital or developmental anomalies and previous ocular surgery groups were younger than those in the myopia and trauma groups. We observed successful anatomic outcomes both with SB and PPV surgeries, particularly in the myopic group.

In this study, we found that the mean age was $11.63 \pm 4.36$ years in children with RRD. Similar to our findings, previous studies reported a mean age ranging from 9.6 to 12.0 years $(10,12-14)$. Among the study groups, children with RRD were older in the myopic group and younger in the congenital/developmental anomaly group. It is plausible that cases in the developmental anomaly group presented at an earlier age than the traumatic and myopia group due to comorbidities. In accordance with previous studies on paediatric RRD, the male gender was also the dominant gender in this study $(5,12,14)$. This preponderance is likely to be associated with the different and adventurous play habits of boys compared to girls (4). Trauma and myopia are the most commonly identified predisposing factors in this study group. Many studies have reported trauma as the leading cause of paediatric RRD, while others reported that myopia was the most common predisposing factor $(10,12,14,15)$. The high rate of trauma in the current study may be related to the proficiency of our clinic as a tertiary referral centre. Furthermore, no specific threshold value was determined to define pathological myopia in children. In accordance with previous studies, we identified myopia as the causative factor when the refractive error was more than -4 diopters. Among etiologic factors studied, trauma is a preventable risk factor for RRD. Sports and game-related accidents are common in the paediatric age group, which may also lead to open globe injuries and consequent severe vision loss (16). Based on these results, we speculated that there might be a need to raise awareness in families and identify factors that may cause eye injury.

At initial presentation, the mean VA was better in the myopic group compared to the other groups with lower rates of PVR and macular involvement. The lack of subjective complaints and the difficulties in the examination of children may lead to a delay in the diagnosis of retinal detachment in the paediatric age group. Macular involvement, PVR development, and chronicity findings are common in paediatric RRD due to late diagnosis (7-9). In this study, myopic patients, whose average patient age is older, might have noticed their visual impairment in the early stages of the disease and were treated earlier compared to others. In contrast to our findings, Gurler et al. (17) evaluated the clinical characteristics of paediatric RRDs and observed no significant difference between trauma and myopia groups with regard to macular involvement and PVR rates. However, their sample size was smaller compared to our study.

SB may be a good option for the initial approach in the management of paediatric RRD. Prior to routine vitrectomy, the success rates of SB is around $70-80 \%(13,18,19)$. In the current study, SB was the most preferred procedure in the myopia group for initial surgery. Myopic cases are a good candidate for primary SB applications compared to traumatic paediatric RRDs. PPV is commonly employed for cases with undetected or multiple retinal tears, and PVR (20). In contrast to adult RRD patients, the posterior vitreous is not detached in paediatric patients. During vitrectomy, complete dissection of the hyaloid from the retina is a complicated process. In such cases, combining scleral band with PPV surgery may be an appropriate option (18). In the trauma group, which has a higher PVR rate compared to the myopia group, PPV was the most preferred surgical technique in the study. The commonly used vitreous substitute for the tamponade effect was silicone oil. Since it is difficult to keep children in a prone position after vitrectomy, silicon oil is commonly used for tamponade in 
paediatric patients undergoing PPV. In addition, silicone oil is associated with lower PVR rates in RRD treatment.

After the initial surgery, higher success rates were observed in both the myopic and previous history ocular surgery groups compared to the other two groups. However, it should be considered that there are only three patients in the latter group. In previous studies, the success rate of single surgery for RRD was reported to be between 52 and $88 \%$ in the paediatric population (11,21-23). After consecutive surgeries to obtain retinal attachment, anatomic success was achieved in both the myopia and trauma groups with $78.7 \%$ and $75.0 \%$, respectively. The number of operations required to demonstrate these results were lower in the myopia group. In the myopia group, SB procedure provided favourable anatomic outcomes and also required fewer operations. Since SB surgery usually does not require tamponade use, there was no need to perform a consecutive surgery for silicone removal. Final anatomic success was significantly better in the myopic group compared to non-myopic cases. Similarly, Barth et al. (14) found the reattachment rate was highest in the myopic and lowest in the ocular abnormalities group. They suggested that surgery in children with ocular abnormalities may be more challenging due to strong retinal adhesions and unusual vitreous liquefaction. In the current study, congenital and developmental disorders and a history of a previous surgery were also found to be a predictive factors for poor final anatomic outcomes. In addition to the afore mentioned anatomic alterations in eyes with ocular abnormalities, the presence of coexisting systemic abnormalities may delay and hamper the evaluation, diagnosis and treatment procedures of these patients. Other factors found relating to poor final anatomic outcomes were young age and macular involvement. Smith et al. (7) noted that younger patients are more likely to demonstrate RRD involving the macula, but no significant difference was found in successful reattachment between age groups. In accordance with our findings, Chang et al. (24) showed that a younger age and macular detachment were associated with poor anatomic outcomes. Moreover, they also found vitrectomy is associated with poor anatomic outcomes when performed as initial procedure. In another study, Wadhwa et al. (12) showed that primary vitreoretinal surgery was associated with poor visual outcomes compared to scleral buckling. Contrary to these findings, we observed that the type of initial surgery was not associated with poor anatomic outcomes in regression analysis. The discrepancy between studies may be due to different sample cohorts, settings and surgical approaches. Although anatomic success was obtained in most of the RD cases, the mean VA was only slightly improved after surgical interventions. Rejdak et al. (25) reported unsatisfactory visual outcomes after vitrectomy in paediatric retinal detachment with PVR. Previous studies regarded PVR as a predictive factor for poor visual outcomes $(11,12,14)$. Trauma was the leading etiological factor and PVR was not uncommon (38\%) among the overall study group. In addition to etiology, other factors such as coexisting ocular anomalies, amblyopia treatment, intraoperative and postoperative complications may also affect visual improvement in paediatric RRD.

\section{Study Limitations}

The current study is limited by its retrospective nature and relatively small study cohort. Since our clinic is a tertiary referral centre, this study population may also not represent the general paediatric population for RRD. The lack of data regarding the perioperative and postoperative VA hampered further comparison analyses based on visual outcomes among groups.

\section{Conclusion}

RRD in children is a significant problem that causes permanent visual impairment. This condition is likely to have a negative effect on the child's family and social life, as well as their future occupation and income. Although associated with poor visual outcomes, careful ophthalmologic assessment and surgical management can maintain sight and impact these young patients' lives positively. Favourable outcomes may be achieved with appropriate surgical intervention after considering the ocular characteristics and clinical findings of the patient.

\section{Ethics}

Ethics Committee Approval: The study was approved by the Hamidiye Ethics Committee of the University of Health Sciences and conducted in adherence to the tenets of the Declaration of Helsinki.

Informed Consent: Retrospective study.

Peer-review: Externally peer-reviewed.

\section{Authorship Contributions}

Concept: Ö.A., Data Collection or Processing: G.G., A.T., Analysis or Interpretation: G.D., Writing: Ş.Ö.

Conflict of Interest: No conflict of interest was declared by the authors.

Financial Disclosure: The authors have no financial relationships relevant to this article to disclose. 


\section{References}

1. Rumelt S, Sarrazin L, Averbukh E, Halpert M, Hemo I. Paediatric vs adult retinal detachment. Eye (Lond) 2007; 21:1473-8.

2. Nagpal M, Nagpal K, Rishi P, Nagpal PN. Juvenile rhegmatogenous retinal detachment. Indian J Ophthalmol 2004; 52:297-302.

3. Nuzzi R, Lavia C, Spinetta R. Paediatric retinal detachment: a review. Int I Ophthalmol 2017; 10:1592-603.

4. Lee RW, Mayer E), Markham RH. The aetiology of paediatric rhegmatogenous retinal detachment: 15 years experience. Eye (Lond) 2008; 22:636-40.

5. Eibenberger K, Sacu S, Rezar-Dreindl S, Schmidt-Erfurth U, Stifter E, Georgopoulos M. Clinical characteristics and surgical outcome of pediatric and early adulthood retinal detachment [published online ahead of print, 2020 Mar 19]. Eur ) Ophthalmol 2020; 1120672120913030. doi:10.1177/1120672120913030

6. Drenser K. Pearls for managing pediatric retinal detachments. Ophthalmology 2019; 126:1271-2.

7. Smith JM, Ward LT, Townsend JH, et al. Rhegmatogenous Retinal Detachment in Children: Clinical Factors Predictive of Successful Surgical Repair. Ophthalmology 2019; 126:1263-70.

8. Weinberg DV, Lyon AT, Greenwald MJ, Mets MB. Rhegmatogenous retinal detachments in children: risk factors and surgical outcomes. Ophthalmology 2003; 110:1708-13.

9. Wenick AS, Barañano DE. Evaluation and management of pediatric rhegmatogenous retinal detachment. Saudi I Ophthalmol 2012; 26:255-63.

10. Fivgas $G D$, Capone A Jr. Pediatric rhegmatogenous retinal detachment. Retina 2001; 21:101-6.

11. Gonzales CR, Singh S, Yu F, Kreiger AE, Gupta A, Schwartz SD. Pediatric rhegmatogenous retinal detachment: clinical features and surgical outcomes. Retina 2008; 28:847-52.

12. Wadhwa N, Venkatesh P, Sampangi R, Garg S. Rhegmatogenous retinal detachments in children in India: clinical characteristics, risk factors, and surgical outcomes. J AAPOS 2008; 12:551-4.

13. Errera MH, Liyanage SE, Moya R, Wong SC, Ezra E. Primary scleral buckling for pediatric rhegmatogenous retinal detachment. Retina 2015; 35:1441-9.
14. Barth T, Zeman F, Helbig H, Oberacher-Velten I. Clinical Features and Outcome of Paediatric Retinal Detachment. Ophthalmologica 2017; 237:63-72.

15. Wang NK, Tsai $\mathrm{CH}$, Chen $\mathrm{YP}$, et al. Pediatric rhegmatogenous retinal detachment in East Asians. Ophthalmology 2005; 112:1890-5.

16. Şahin Atik S. Open Globe Injury in Children. J Pediatr Res 2020; 7:77-80.

17. Gurler B, Coskun E, Öner V, Comez A, Erbagci I. Clinical characteristics and surgical outcomes of pediatric rhegmatogenous retinal detachment. Int Ophthalmol 2016; 36:521-5.

18. Hudson JR. Retinal detachments in children. Trans Ophthalmol Soc U K 1965;85:79-91.

19. Okinami S, Ogino N, Nishimura T, Tano Y. Juvenile Retinal Detachment. Ophthalmologica 1987; 194:95-102.

20. Znaor L, Medic A, Binder S, Vucinovic A, Marin Lovric J, Puljak L. Pars plana vitrectomy versus scleral buckling for repairing simple rhegmatogenous retinal detachments. Cochrane Database Syst Rev 2019;3:CD009562. Published 2019 Mar 8. doi:10.1002/14651858.CD009562.pub2

21. Rahimi M, Bagheri M, Nowroozzadeh MH. Characteristics and outcomes of pediatric retinal detachment surgery at a tertiary referral center. J Ophthalmic Vis Res 2014; 9:210-4.

22. Winslow RL, Tasman W. Juvenile rhegmatogenous retinal detachment. Ophthalmology 1978; 85:607-18.

23. Akabane N, Yamamoto S, Tsukahara I, et al. Surgical outcomes in juvenile retinal detachment. Jpn J Ophthalmol 2001; 45:40911.

24. Chang PY, Yang $\mathrm{CM}$, Yang $\mathrm{CH}$, et al. Clinical characteristics and surgical outcomes of pediatric rhegmatogenous retinal detachment in Taiwan. Am J Ophthalmol 2005; 139:1067-72.

25. Rejdak R, Nowakowska D, Wrona K, Maciejewski R, Junemann AG, Nowomiejska K. Outcomes of Vitrectomy in Pediatric Retinal Detachment with Proliferative Vitreoretinopathy. I Ophthalmol 2017; 2017:8109390. 